\title{
THE SETTLING-TIME REDUCIBILITY ORDERING
}

\author{
BARBARA F. CSIMA AND RICHARD A. SHORE
}

\begin{abstract}
To each computable enumerable (c.e.) set $A$ with a particular enumeration $\left\{A_{s}\right\}_{s \in \omega}$, there is associated a settling function $m_{A}(x)$, where $m_{A}(x)$ is the last stage when a number less than or equal to $x$ was enumerated into $A$. One c.e. set $A$ is settling time dominated by another set $B\left(B>>_{s t} A\right)$ if for every computable function $f$, for all but finitely many $x, m_{B}(x)>f\left(m_{A}(x)\right)$. This settling-time ordering, which is a natural extension to an ordering of the idea of domination, was first introduced by Nabutovsky and Weinberger in [3] and Soare [6]. They desired a sequence of sets descending in this relationship to give results in differential geometry. In this paper we examine properties of the $<_{s t}$ ordering. We show that it is not invariant under computable isomorphism, that any countable partial ordering embeds into it, that there are maximal and minimal sets, and that two c.e. sets need not have an inf or sup in the ordering. We also examine a related ordering, the strong settling-time ordering where we require for all computable $f$ and $g$, for almost all $x, m_{B}(x)>f\left(m_{A}(g(x))\right)$.
\end{abstract}

August 22, 2006

\section{INTRODUCTION}

This paper concerns the settling-time reducibility ordering on c.e. sets, which we now define.

Definition 1.1. (i) For every computably enumerable (c.e.) set $W_{e}$ and associated enumeration $W_{e, s}$,we define the settling (or modulus) function: $m_{e}(x)=(\mu s)\left[W_{e, s}\left\|x=W_{e}\right\| x\right]$ where $A \| x=\{y \leq x \mid y \in A\}$.

(ii) For c.e. sets $A$ and $B$, we say $A$ settling time dominates $B$ and write $A>_{s t} B$ iff $\left(\exists W_{i}=A\right)\left(\exists W_{j}=B\right)$

$$
(\forall \text { computable } f)(\text { a.e. } x)\left[m_{i}(x)>f\left(m_{j}(x)\right)\right] .
$$

(We use "a.e. $x$ " to mean "for all but finitely many $x$ ".) Andre Nies showed that (ii) is equivalent to $\left(\forall W_{i}=A\right)\left(\forall W_{j}=B\right)$ [(1) holds]. Hence we will abuse notation by writing $m_{A}$ for $m_{e}$ when $A=W_{e}$. We denote the structure of the c.e. sets with the relation $<_{s t}$ as $\mathcal{E}_{s t}$.

This ordering was first introduced by Nabutovsky and Weinberger [3] and Soare [6]. Nabutovsky and Weinberger desired a descending sequence in $\mathcal{E}_{s t}$ to use in the construction of various manifolds that gave information

Partially supported by Canadian NSERC Discovery Grant 312501.

Partially supported by NSF Grants DMS-0100035 and DMS-0554855. 
on the geometry of Riemannian metrics modulo diffeomorphisms. Soare constructed such a sequence as described in [6]. Sequences with additional useful or interesting properties were constructed by Csima [1] and Csima and Soare [2]. For general background information on the ordering and its applications to differential geometry we suggest Soare [6], Csima and Soare [2] and Weinberger [8].

In this paper we investigate properties of the $<_{s t}$ relation and order theoretic facts about $\mathcal{E}_{s t}$. We show (Theorem 2.1) that the relation itself is not invariant under computable isomorphism. On the other hand, in $\S 7$, we examine a stronger relation $<_{s s t}$ introduced in [2] and show that it is invariant under not only computable isomorphism but even under $w t t$-degree. We also construct a descending chain in this ordering to answer a question from [2]. As for the structure of $\mathcal{E}_{\text {st }}$, we show (Theorem 3.1) that any countable partial ordering embeds into it; it has maximal (Theorem 4.1) and minimal (Theorem 5.1) elements; and that there are pairs of c.e. sets with no inf and ones with no sup (Theorem 6.2).

For Computability Theory, we follow the notation of Soare's Recursively enumerable sets and Degrees [5] and new notation from Soare's Computability Theory and Applications [7], which we also define.

We let $\left\{W_{e, s}\right\}_{e, s \in \omega}$ be any standard enumeration of the c.e. sets. We let $\varphi_{e}$ denote the $e$-th partial computable function according to some standard enumeration. We often use the convention that $\varphi_{e, s}(n) \downarrow \Rightarrow(\forall m \leq n) \varphi_{e, s}(m) \downarrow$, i.e. that the partial computable functions converge on numbers in order.

We write " $\forall \infty$ " and "for a.e. $x$ " for "for all but finitely many $x$ ". Similarly, " $\exists \infty x$ " denotes "there are infinitely many $x$ ".

\section{NoninVARIANCE Under COMPUTABle ISOMORPHism}

Nies (unpublished, see [2]) showed that the settling time ordering is welldefined on c.e. sets. Csima and Soare [2] show that it is not well-defined on Turing degrees. In fact, the settling time ordering is not invariant under computable isomorphism.

Theorem 2.1. There exist c.e. sets $A$ and $B$ such that $A \equiv_{1} B$ but $A>{ }_{s t} B$.

Proof. We first recursively associate to each $n \in \omega$ an even $x_{n} \in \omega$ in the following way. Let $x_{0}=0$. For $n \geq 0$, let $x_{n+1}=x_{n}+2(n+1)$.

We now construct the sets $A$ and $B$ by stages.

Stage s: Step 1. Enumerate $x_{s}$ into $A$ (to ensure that $A$ will be infinite).

Step 2. If $x_{n+1}$ was enumerated into $B$ at stage $t<s$ and $\varphi_{i}(t)$ converged at stage $s$ for some $i<n$, enumerate $x_{n}+2(i+1)$ into $A$ (note that this is the only circumstance under which $x_{n}+2(i+1)$ will be enumerated into $A$ ).

Step 3. If $2 m$ is enumerated into $A$, enumerate $x_{m}$ into $B$.

Lemma 2.2. $A \leq_{1} B$.

Proof. Let $f(2 m)=x_{m}$ and $f(2 m+1)=x_{m}+1$. By construction $2 m \in A$ iff $x_{m} \in B$. Also, there are no odd numbers in $A$, and there are only numbers 
of the form $x_{m}$ in $B$. Thus $n \in A$ iff $f(n) \in B$. It is also clear that $f$ is one to one and computable.

Lemma 2.3. $B \leq_{1} A$.

Proof. Let $g(m)=2 n$ if $m=x_{n}$, and $g(m)=2 m+1$ otherwise. Then $g$ is a computable one to one function such that $m \in B$ iff $g(m) \in A$.

Lemma 2.4. $A>_{s t} B$.

Proof. Let $f$ be a total computable function. We must show that $\left(\forall^{\infty} x\right) m_{A}(x)$ $>f\left(m_{B}(x)\right)$. It suffices to show that for a.e. $x$, if $x$ entered $B$ at a stage $s$, some $z \leq x$ entered $A$ at a stage greater than $f(s)$. Let $i$ be such that $f=\varphi_{i}$. Let $x>x_{i+1}$, and suppose $x$ entered $B$ at stage $s$. Then by construction, $x=x_{n+1}$ for some $n>i$. At the stage $t$ when $\varphi_{i}(s)$ converged, $x_{n}+2(i+1)$ was enumerated into $A$. Note that $f(s)=\varphi_{i}(s)<t$, and that $x_{n}+2(i+1)<x_{n+1}$.

Thus $A \equiv_{1} B$ and $A>_{s t} B$.

As $A \equiv_{1} B$ if and only if there is a computable permutation $p$ of $\mathbb{N}$ such that $B$ is the image of $A$ under $p$, the relation $<_{s t}$ is not invariant even under computable permutations (isomorphisms) of $\mathbb{N}$. From the viewpoint of computability theory presented by Rogers [4, Ch. 4], one can question if such a relation is properly viewed as computability theoretic. In $\S 7$ we study a stronger notion $<_{\text {sst }}$ also described in [2] which is invariant under computable permutations and more and construct the descending chain desired by Nabutovsky and Weinberger in that ordering as asked for in [2].

\section{Embedding Partial Orderings}

Theorem 3.1. Consider a computable partial ordering $P$ on $\mathbb{N}$. We build a uniformly c.e. sequence $\left\{A_{n}\right\}$ of sets such that $A_{i}<_{s t} A_{j} \Longleftrightarrow i<_{P}$ $j$. Hence, by the existence of a computable universal partial ordering, any countable partial ordering can be embedded into $\mathcal{E}_{\text {st }}$.

Proof. We construct the sequence $\left\{A_{n}\right\}$ by stages. At each stage $s$ there will be only finitely many numbers enumerated.

If $i<_{P} j$ we wish to ensure that $\varphi_{n}$ total $\Rightarrow\left(\forall^{\infty} x\right) m_{A_{j}}(x)>\varphi_{n} \circ m_{A_{i}}(x)$. We do this by observing the following Golden Rule.

Golden Rule. If $i<_{P} j$ and $\langle i, j\rangle \leq s$, then at stage $s$ we enumerate $x$ into $A_{i}$ only if there are appointed $x_{0}, \ldots, x_{s}$, all less than $x$, and we make the commitment that $x_{n}$ will be enumerated into $A_{j}$ at the stage when $\varphi_{n}(s) \downarrow$, should this ever happen. We refer to the $x_{n}$ as "guards" ready to enter $A_{j}$ in case $\varphi_{n}$ converges on $s$.

If $i \nless_{P} j$ we wish to ensure that $\left(\exists^{\infty} x\right) m_{A_{i}}(x) \geq m_{A_{j}}(x)$. At each stage $s$ with $\langle i, j\rangle\left\langle s\right.$, we will obtain a witness $n_{s}$ and we will promise to keep $m_{A_{i}}\left(n_{s}\right) \geq m_{A_{j}}\left(n_{s}\right)$ by enumerating into $A_{i}$ below $n_{s}$ whenever we enumerate into $A_{j}$ below $n_{s}$. 
Stage s: For each $n=\langle i, j\rangle<s$ such that $i \nless_{P} j$, do as follows, in turn.

Our plan is to choose an $n_{s}$, enumerate it into $A_{i}$, and ensure that no $y \leq n_{s}$ enters $A_{j}$ at $t \geq s$ unless some $z<n_{s}$ enters $A_{i}$ at $t$. To find $n_{s}$, note first that there are only finitely many elements that may enter $A_{j}$ due to prior commitments, say $m$ many. Now looking at $P \uparrow s \times s$, there are finitely many finite maximal chains of the form $k_{0}=i<_{P} k_{1}<_{P} \ldots<_{P} k_{r}$. Note that $j$ does not appear in any of these chains. Choose $m+1$ numbers that are ready to enter $A_{i}$ (should something enter $A_{j}$ ) such that there are enough guards for $\varphi_{0}, \ldots, \varphi_{s}$ for all $P$-chains. That is, at stage $s$ we appoint finitely many possible entrants of sets, all greater than any number mentioned earlier in the construction, according to the following rules. We appoint $z_{1}, \ldots, z_{m}<n_{s}$ and promise to enumerate $z_{k}$ into $A_{i}$ at the stage when the $k^{t h}$ number less than or equal to $n_{s}$ is enumerated into $A_{j}$. For each $P$-chain of the form $k_{0}=i<_{P} k_{1}<_{P} \ldots<_{P} k_{r}$, and each pair $\left(k_{p}, k_{q}\right)$ with $0 \leq p<q \leq r$, for each possible entrant $y$ of $A_{k_{p}}$ appointed at this stage, we appoint $y_{0}^{\left(k_{p}, k_{q}\right)}, \ldots, y_{s}^{\left(k_{p}, k_{q}\right)}<y$ and promise to enumerate $y_{k}^{\left(k_{p}, k_{q}\right)}$ into $A_{k_{q}}$ at the stage when $\varphi_{k}\left(s\left(y, k_{p}\right)\right) \downarrow$, where $s\left(y, k_{p}\right)$ denotes the stage when $y$ enters $A_{k_{p}}$. Note that since $j$ doesn't appear in any of the $P$-chains, this won't cause the number of possible future entrants of $A_{j}$ to go up. Now we enumerate $n_{s}$ into $A_{i}$.

Lemma 3.2. If $i<_{P} j$ then $A_{i}<_{s t} A_{j}$.

Proof. Suppose $i<_{P} j$. Suppose $f$ is a total function. We must show $\forall^{\infty} x\left(m_{A_{j}}(x)>f\left(m_{A_{i}}(x)\right)\right)$. Let $k$ be such that $f=\varphi_{k}$. Let $s=\langle i, j\rangle$. By stage $s$, we have appointed only finitely many possible entrants of $A_{i}$. Let $t \geq k$ be a stage by which all of those that were appointed before stage $s$ and belong to $A_{i}$ entered $A_{i}$. Then any $x$ which entered $A_{i}$ after stage $t$ was appointed after stage $s$, so the $P$-chain containing $i$ and $j$ was considered, and an $x_{k}^{(i, j)}<x$ was appointed to enter $A_{j}$ at the stage when $\varphi_{k}(s(x, i)) \downarrow$.

Lemma 3.3. If $i \nless_{P} j$ then $A_{i} \nless_{s t} A_{j}$.

Proof. Suppose $i \nless_{P} j$. Let $n=\langle i, j\rangle$. At every stage $s>n$, an $n_{s}$ was enumerated into $A_{i}$, and for every $x \leq n_{s}$ that entered $A_{j}$ after stage $s$, something less than $n_{s}$ entered $A_{i}$. So $m_{A_{i}}\left(n_{s}\right) \geq m_{A_{j}}\left(n_{s}\right)$, so $A_{i} \nless_{s t} A_{j}$.

By the above lemmas, the sequence $\left\{A_{n}\right\}$ has the required properties.

One anomaly about $\mathcal{E}_{s t}$ relative to other partial orderings studied in computability theory is that it is a strict partial ordering rather than a reflexive one. This has the seemingly strange consequence that an embedding of, for example, an incomparable pair $x, y$ can be realized by any set $A$ as the image for both $x$ and $y$ (or $A$ and any finite variation of $A$ ). The largest equivalence relation on $\mathcal{E}_{s t}$ (or any strict partial order) that respects the given ordering and gives a reflexive partial ordering as a quotient is given by $A \equiv_{s t} B \Leftrightarrow\left\{C \mid C>_{s t} A\right\}=\left\{C \mid C>_{s t} B\right\} \&\left\{C \mid C<_{s t} A\right\}=\left\{C \mid C<_{s t} B\right\}$. 
One could instead of $\mathcal{E}_{s t}$ then reasonably study its quotient $\mathcal{E}_{s t}^{*}$ by this equivalence relation with the natural partial ordering $\leq$. It is easy to see, however, that by simply adding on extra elements to a given partial ordering $P$ one can produce one $P^{\prime}$ such that any embedding of $P^{\prime}$ into $\mathcal{E}_{s t}$ will restrict to one of $P$ into $\mathcal{E}_{s t}^{*}$. Thus every countable partial ordering can be embedded in $\mathcal{E}_{s t}^{*}$ as well.

\section{A Maximal Set}

Theorem 4.1. There exists an $A$ such that for all $e, W_{e} \ngtr$ st $A$.

Proof. We will construct a c.e. set $A$ by stages. For each $e$, we wish to ensure that $W_{e} \ngtr_{s t} A$. That is, we must ensure that there is a computable function $f$ and infinitely many "winning locations" $x$ such that $f\left(m_{A}(x)\right)>$ $m_{e}(x)$. We will do this by having separate strategies for each $e$ which do not interfere with one another. Note that the computable functions providing the counterexample for each $e$ do not have to be found effectively in $e$.

We first outline the strategy for defeating $W_{0}$. We allow ourselves to enumerate even numbers for the sake of defeating $W_{0}$. We save the odds for other $W_{e}$.

To show $W_{0} \ngtr_{s t} A$, we need a computable function $f$ and infinitely many $x$ such that $f \circ m_{A}(x) \geq m_{0}(x)$.

If $W_{0}$ is finite, we will win with the identity, by making $A$ infinite (this will happen automatically, as an infinite $W_{e}$ will cause infinitely many numbers to enter $A$ ).

If $W_{0}$ is infinite, $\Gamma_{0}, \Gamma_{1}, \ldots$ will mark the winning locations.

We will define a partial computable function $f$. We will either win with the identity, or else $f$ will be almost total, and we will win with a finite variation of $f$.

Start off with all $\Gamma_{k}$ undefined. We let $\Gamma_{k}[s]$ denote the position of the marker $\Gamma_{k}$ at the end of stage $s$, and let $\Gamma_{k}$ denote the final position of the marker, i.e., $\Gamma_{k}=\lim _{s} \Gamma_{k}[s]$. We assume that $\Gamma_{k}[s+1]=\Gamma_{k}[s]$ unless the construction states otherwise.

Stage $s+1$ : Suppose $x$ is enumerated into $W_{0}$ at stage $s$.

Case 1: $\Gamma_{k-1}[s]<x \leq \Gamma_{k}[s]$ for some $k$. Let $\Gamma_{l}[s+1]$ be undefined for $l>k$. If a $y$ with $\Gamma_{k-1}[s]<y \leq \Gamma_{k}[s]$ has already been enumerated into $A$ at a stage $y_{A}$ with $f\left(y_{A}\right)$ not yet defined, define $f\left(y_{A}\right)=s+1$. Otherwise, enumerate an even number $y, \Gamma_{k-1}[s]<y \leq \Gamma_{k}[s]$ into $A$.

Case 2: $\Gamma_{k}[s]<x$, and $\Gamma_{l}[s]$ is undefined for $l>k$. If there is a $y>\Gamma_{k}[s]$ that entered $A$ at stage $y_{A}$ with $f\left(y_{A}\right)$ undefined, set $f\left(y_{A}\right)=s+1$. If there is no such $y$, set $\Gamma_{k+1}[s+1]$ to be an even number greater than $x$ and greater than any number enumerated into $A$ so far, and enumerate $\Gamma_{k+1}[s+1]$ into A.

If we did not enumerate anything into $A$ at stage $s$, define $f(s)=s$. 
If $W_{0}$ is infinite then eventually all the $\Gamma_{k}$ will be defined and reach a limit. Note also that by construction, $m_{0}\left(\Gamma_{k-1}\right)<m_{0}\left(\Gamma_{k}\right)$, since whenever a number less than or equal to $\Gamma_{k-1}[s]$ enters $W_{0, s+1}, \Gamma_{k}[s+1]$ is undefined, and $\Gamma_{k}[t+1]$ is only redefined at a later stage $t+1$ (to be larger than any previously mentioned number) if a number greater than $\Gamma_{k-1}[t]$ enters $W_{e, t+1}$.

In each interval $\left(\Gamma_{k-1}, \Gamma_{k}\right]$, if some $x$ entered $W_{0}$, we either enumerated into $A$ in that interval, or defined $f$ on a stage number of something that had already been enumerated into $A$ in that interval. This is because if at stage $s$ we act to define $\Gamma_{l}[s]$, then we only do this if $\Gamma_{m}[s-1]$ is defined for all $m<l$, and we define $\Gamma_{l}[s]$ to be larger than any number previously mentioned in the construction. Hence there will exist a $k \leq l$ such that $\left(\Gamma_{l-1}[s], \Gamma_{l}[s]\right] \subseteq$ $\left(\Gamma_{k-1}, \Gamma_{k}\right]$; and whenever $x$ enters $W_{0, s+1}$, if $\Gamma_{l-1}[s]<x \leq \Gamma_{l}[s]$ then we either enumerate into $A$ or define $f$ on a stage number of something that had already been enumerated into $A$ in the interval $\left(\Gamma_{l-1}[s], \Gamma_{l}[s]\right]$, and if $x>\Gamma_{l}[s]$ with $\Gamma_{l+1}[s]$ undefined, we either define and enumerate $\Gamma_{l+1}[s+1]$ into $A$, or we define $f$ on the stage number when a number $y>\Gamma_{l}[s]$ entered $A$. In the latter case, if $W_{0}$ is infinite, both $x$ and $y$ are in an interval $\left(\Gamma_{m}[t], \Gamma_{m+1}[t]\right]$ for some $t>s+1$ and $m \leq l$.

Let $A_{[0]}$ denote the part of $A$ that we are controlling for $W_{0}$ (in this case the even numbers). Note that for any $x, m_{A_{[0]}}(x) \leq m_{A}(x)$.

If there are infinitely many intervals where the last action was to enumerate into $A$, then on these intervals we have $m_{0}\left(\Gamma_{k}\right) \leq m_{A_{[0]}}\left(\Gamma_{k}\right) \leq m_{A}\left(\Gamma_{k}\right)$. So $W_{0}$ is defeated. If not, then on almost all intervals our last action was to define $f$ on a stage number. So $f$ is almost total, and $m_{0}\left(\Gamma_{k}\right) \leq f\left(m_{A_{[0]}}\left(\Gamma_{k}\right)\right)$ for almost all $k$. Let $f^{*}$ be an increasing computable function with $f^{*}(x) \geq$ $f(x)$ for almost all $x$. Then $m_{0}\left(\Gamma_{k}\right) \leq f\left(m_{A_{[0]}}\left(\Gamma_{k}\right)\right) \leq f^{*}\left(m_{A_{[0]}}\left(\Gamma_{k}\right)\right) \leq$ $f^{*}\left(m_{A}\left(\Gamma_{k}\right)\right)$.

So we see that not only have we defeated $W_{0}$, but it doesn't matter what we enumerate into $A$ on the odd numbers.

To defeat $W_{1}$ we will work with numbers of the form $1+4 n$. We will have to use three partial computable functions $f_{1}, f_{2}, f_{3}$. In this case we will first enumerate into $A$, then define $f_{1}$ on the stage number, then $f_{2}$, and then $f_{3}$, before enumerating into $A$ again. This way, if we don't win on the identity, $f_{1}$ is almost total. If we don't win on $f_{1}$, then $f_{2}$ is almost total, and so forth.

We now outline the general case. To defeat $W_{e}$, we work with numbers of the form $2^{e}-1+2^{e+1} n$. That is, for each $W_{e}$, we enumerate a set $A_{[e]} \subseteq \omega_{[e]}$, where $\omega_{[e]}=\left\{2^{e}-1+2^{e+1} n \mid n \in \omega\right\}$. Note that if $e \neq k$ then $\omega_{[e]} \cap \omega_{[k]}=\emptyset$.

We will build partial computable functions $f_{1}^{e}, f_{2}^{e}, \ldots, f_{2^{e+1}-1}^{e}$.

As before, if $W_{e}$ is infinite, $\Gamma_{0}^{e}, \Gamma_{1}^{e}, \ldots$ will mark the winning locations.

Start with all $\Gamma_{k}^{e}$ undefined.

Stage s: Suppose $x$ is enumerated into $W_{e}$ at stage $s$. 
Case 1: $\Gamma_{k-1}^{e}[s]<x \leq \Gamma_{k}^{e}[s]$ for some $k$. Let $\Gamma_{l}^{e}[s+1]$ be undefined for $l>k$. If some $y$ with $\Gamma_{k-1}^{e}[s]<y \leq \Gamma_{k}^{e}[s]$ was enumerated into $A_{[e]}$ at a stage $y_{A}$ with $f_{m}\left(y_{A}\right)$ undefined for some $m$, let $m$ be the least such, and define $f_{m}\left(y_{A}\right)=s$. Otherwise, enumerate a number $y \in \omega_{[e]}, \Gamma_{k-1}^{e}[s]<y \leq \Gamma_{k}^{e}[s]$ into $A_{[e]}$.

Case 2: $\Gamma_{k}^{e}[s]<x$, and $\Gamma_{l}^{e}[s]$ is undefined for $l>k$. If there is an $m$ and a $y>\Gamma_{k}^{e}[s]$ which was enumerated into $A_{[e]}$ at a stage $y_{A}$ with $f_{m}^{e}\left(y_{A}\right)$ now undefined, choose the least such $m$ and define $f_{m}^{e}\left(y_{A}\right)=s$. If there is no such $m$ and $y$, set $\Gamma_{k+1}^{e}[s+1]$ to be in $\omega_{[e]}$ and to be greater than $x$ and greater than any number enumerated into $A_{[e]}$ so far. Enumerate $\Gamma_{k+1}^{e}[s+1]$ into $A_{[e]}$.

If we did not enumerate anything into $A$ at stage $s$, define $f_{m}^{e}(s)=s$ for all $1 \leq m \leq 2^{e+1}-1$.

If $W_{e}$ is infinite then eventually all the $\Gamma_{k}^{e}$ will be defined and reach a limit. Note also that by construction, $m_{e}\left(\Gamma_{k-1}^{e}\right)<m_{e}\left(\Gamma_{k}^{e}\right)$.

In each interval $\left(\Gamma_{k-1}, \Gamma_{k}\right]$, if some $x$ entered $W_{e}$, we either enumerated into $A$ in that interval, or defined $f_{m}$ on a stage number of something that was enumerated into $A$ in that interval for the least $m$ for which $f_{m}$ was undefined on a stage number of something already enumerated in that interval. This is because, as in the the discussion for $W_{0}$, any interval that is ever defined can only change by being absorbed by a larger interval. The fact that even in the limit interval it is still the least $m$ for which $f_{m}$ was undefined on a stage number of something enumerated into the interval that gets defined follows from the fact that an interval is only ever set by enumerating it's right endpoint into $A$.

Let $f_{0}$ denote the identity function.

Let $m$ be least such that there are infinitely many intervals where our last action was to define $f_{m}$. Note that $m$ exists by the Pigeon Hole Principle. By our convention of first trying to define $f_{m}$ for the least $m \geq 1$, and by the minimality of $m, f_{m}$ is almost total. Let $f^{*}$ be a total computable increasing function such that $f_{m}(x) \leq f^{*}(x)$ for all $x$ on which $f_{m}$ is defined. Then for those intervals $\left(\Gamma_{k-1}, \Gamma_{k}\right]$ where our last action was to define the $f_{m}$ we have $m_{e}\left(\Gamma_{k}\right) \leq f_{m}\left(m_{A_{[e]}}\left(\Gamma_{k}\right)\right) \leq f^{*}\left(m_{A_{[e]}}\left(\Gamma_{k}\right)\right) \leq f^{*}\left(m_{A}\left(\Gamma_{k}\right)\right)$.

Of course, any set maximal in $\mathcal{E}_{s t}$ is (i.e. its equivalence class is) maximal in $\mathcal{E}_{s t}^{*}$.

\section{Minimal Sets}

If $A$ and $B$ are c.e. sets such that $A>_{s t} B$, then $A$ must be high (see Csima-Soare [2]). Hence if $A$ is not high, then $A \ngtr_{s t} B$ for any $B$. Thus $A$ is minimal in $\mathcal{E}_{s t}$. However, this is not entirely satisfying since $A$ does not settling time dominate any set at all. We note that if $A>_{s t} C$ for any infinite c.e. set $C$, then $A$ settling time dominates every computable set. (So all the infinite computable sets are in the same class, 0 , in $\mathcal{E}_{s t}^{*}$.) Hence we will write $A>_{s t} 0$ if $A$ settling time dominates any infinite c.e. set. 
In this section we show that there is a nontrivial (i.e. above 0) minimal set in $\mathcal{E}_{s t}$ (and so in $\mathcal{E}_{s t}^{*}$ ). That is, we construct a c.e. set $A$ such that $A>_{s t} 0$, and for any $B$, if $A>_{s t} B$, then for all $W_{e}, B \ngtr_{s t} W_{e}$. Indeed, we will build $A$ such that for all $e$ if $W_{e}$ is noncomputable, then $A \ngtr s t W_{e}$.

Theorem 5.1. There exists a nontrivial minimal set in $\mathcal{E}_{\text {st }}$. Indeed, there exists a c.e. set $A$ such that $A>_{s t} 0$ and if $W_{e}$ is noncomputable then $A \ngtr s t W_{e}$.

Proof. We construct the c.e. set $A$, and also an infinite computable set $C$ such that $A>_{s t} C$. Let $c_{0}=0$ and $c_{n+1}=c_{n}+n+1$. Let $a_{i}^{n}=c_{n}+i$ for $0 \leq i \leq n$. At stage $s$ we enumerate $c_{s}$ into $C$ (so $C$ is computable).

The strategy to make $A>_{s t} C$ will be to try to enumerate $a_{i}^{n}$ into $A$ at a stage after the stage when $\varphi_{i}(n+1) \downarrow$.

For each $W_{e}$, if $W_{e}$ is noncomputable, we want infinitely many $x$ with $m_{e}(x) \geq m_{A}(x)$. Thus, when some $x$ enters $W_{e}$, it would be nice to restrain $A$ below $x$ to ensure that $m_{e}(x) \geq m_{A}(x)$.

For each $e$, we will have restraint functions $r(e, j, s)$ for all $j \geq e$. We will let $R(j, s)=\max \{r(e, j, s) \mid e \leq j\}$.

We now construct $A$ by stages as follows.

Stage $s+1$ : We start off with $\tilde{r}(e, j)=r(e, j, s)$ for all $e, j \in \omega$, and let $\tilde{R}(j)=\max \{\tilde{r}(e, j) \mid e \leq j\}$.

Suppose $x$ is enumerated into $W_{e, s+1}$. If $x<\tilde{r}(e, j)$ for any $j$, reset $\tilde{r}(e, j)=x$ for all such $j$ (note that there can be only finitely many such $j$ ). Otherwise, let $j$ be least such that $\tilde{r}(e, j)=0$, and reset $\tilde{r}(e, j)=x$.

Now, perform the following loop. Suppose $\varphi_{i, s+1}(n+1) \downarrow$ for some $i, n$ with $i \leq n$. If $a_{i}^{n}>\tilde{R}(i)$, enumerate $a_{i}^{n}$ into $A_{s+1}$. For all $e, j$ with $a_{i}^{n}<\tilde{r}(e, j)$, reset $\tilde{r}(e, j)=0$. If anything is enumerated, repeat the loop. Otherwise end the loop and set $r(e, j, s+1)=\tilde{r}(e, j)$ for all $e, j$.

Note that by construction, the only way that $r(e, j, s)$ can increase as a function of $s$ is if the restraint is injured by enumeration into $A$ during a loop and set to 0 , and then reset to a larger number in a later stage. That is, we have $r(e, j, s+1) \leq r(e, j, s)$ unless $r(e, j, s)=0$. Also, if $r(e, j, s+1)>$ $r(e, j, s)=0$, then this was because $r(e, j, s+1)$ was enumerated into $W_{e, s+1}$. Hence for any $e, j \in \omega, \lim _{s} r(e, j, s)<\infty$ or $\lim \sup _{s} r(e, j, s)=\infty$.

Lemma 5.2. For all e, if $W_{e}$ is noncomputable, then $A \ngtr_{s t} W_{e}$.

Proof. Case 1: For all $j \geq e, \lim _{s} r(e, j, s)<\infty$. Let $x_{j}=\lim _{s} r(e, j, s)$. Then either $W_{e}$ is finite, or there exist infinitely many $j$ such that $x_{j}<x_{j+1}$, and for all $j, m_{e}\left(x_{j}\right) \geq m_{A}\left(x_{j}\right)$. To see this suppose that $r(e, j, s)$ reaches its final value $x$ at stage $t$. It must be that $x$ entered $W_{e}$ at stage $t$. If some $y<x$ enters $A$ at a later stage $v$ then $r(e, j, v)=0$ for a contradiction. Thus $A \ngtr s t W_{e}$.

Case 2: Otherwise, let $j$ be the least such that $\lim \sup _{s} r(e, j, s)=\infty$. By our conventions, if $\varphi_{i}$ is not total, then it converges on only finitely many $n$. Hence the function $f(n)=(\mu s)\left[(\forall i \leq j) \varphi_{i}(n) \downarrow \rightarrow \varphi_{i, s}(n) \downarrow\right]$ is computable. 
For $x>\max \left\{\lim _{s} r(e, i, s) \mid i<j\right\}$, we can certainly compute $m$ such that $x<c_{m}$. Then $x \in W_{e} \Longleftrightarrow x \in W_{e, f(m+1)}$ for otherwise we would have $\lim _{s} r(e, j, s) \leq x$. Thus $W_{e}$ is computable.

Lemma 5.3. $A>_{s t} C$.

It suffices to show that for all $i$, if $\varphi_{i}$ is total then there exists an $m$ such that whenever $a_{i}^{n}>m$ and $\varphi_{i, s}(n+1) \downarrow$ then there is a stage $t \geq s$ where $a_{i}^{n}$ is enumerated into $A$.

For $e \leq i$, let $r(e, i)=\lim \sup _{s} r(e, i, s)$. Let $R(i)=\max \{r(e, i) \mid r(e, i)<$ $\infty \wedge e \leq i\}$. Suppose $a_{i}^{n}>R(i)$, and suppose $\varphi_{i, s}(n+1) \downarrow$. For $t \geq s$, let $b(t)=\min \{r(e, i, t) \mid r(e, i, t)>0 \wedge r(e, i)=\infty \wedge e \leq i\}$. We wish to show that there is a stage $t \geq s$ such that $b(t)$ is undefined, and hence $a_{i}^{n}$ would be enumerated into $A_{t}$. The function $b(t)$ cannot increase without being undefined in between. (For $b(v)$ to increase, $r(e, i, v)$ must increase for the $i$ such that $r(e, i, t)$ is the smallest among the relevant values. Before it can increase, however, it must first become 0 because some smaller element entered $A$ and so all the other relevant $r(e, j, v)$ for which $r(e, j, t)$ were larger than $r(e, i, t)$ also become 0 .) So we may assume for a contradiction that $b=\lim _{t} b(t)$ exists. Let $t_{1}$ be such that $b=r\left(e, i, t_{1}\right)$ with $r(e, i)=\infty$, $e \leq i$, and $b(t)=b$ for all $t \geq t_{1}$. Since $\lim \sup _{t} r(e, i, t)=\infty$, there is some number less than $b$ enumerated into $A$ at a stage $t_{2}>t_{1}$. But then $b\left(t_{2}\right)$ is undefined.

\section{NO INFS AND SUPS}

In this section, we show that infs and sups need not exist in $\mathcal{E}_{s t}$ or $\mathcal{E}_{s t}^{*}$.

One way of showing that there is a pair with no sup, is to build a set which is both maximal and minimal in the $<_{s t}$ ordering. We do not do this in detail, since the existence of such a pair follows from the construction we do to show there is a pair with no inf. However, we state the existence of such a set, and outline how to go about it's construction.

Theorem 6.1. There exists a c.e. set which is both maximal and minimal with respect to the $<_{\text {st }}$ ordering.

Proof Sketch. Combine the constructions of the maximal and minimal set $A$. There will be finite injury, since the minimal set construction wishes to hold members out of the set $A$. By introducing an extra function $f_{2^{e+1}}^{e}$ for the $e$-th requirement of the maximal set construction, one can afford to injure the requirements by "kicking the $\Gamma_{k}^{e}$ markers" a distance of $2^{e+1} r$ when a minimality requirement of higher priority wishes to restrain $A$ below $r$.

We now give a detailed construction showing that there are c.e. sets $A$ and $B$ which have no inf in $\mathcal{E}_{\text {st }}$ and ones $C$ and $D$ which have no sup. We do this by also building c.e. sets $E, F, G$ such that $A, B>{ }_{s t} C, D ; E>_{s t} A$ but $E \ngtr s t B ; F>_{s t} D$ but $F \ngtr s t C ; C, D>_{s t} G ;\left.A\right|_{s t} B$ and $\left.C\right|_{s t} D$. We also ensure that there is no c.e. set $H$ such that $A>_{s t} H>_{s t} C$, we refer 
to this property as having a gap between $A$ and $C$ (and indicate it by the double line from $A$ to $C$ in the diagram below). That is, the sets will satisfy the following diagram:

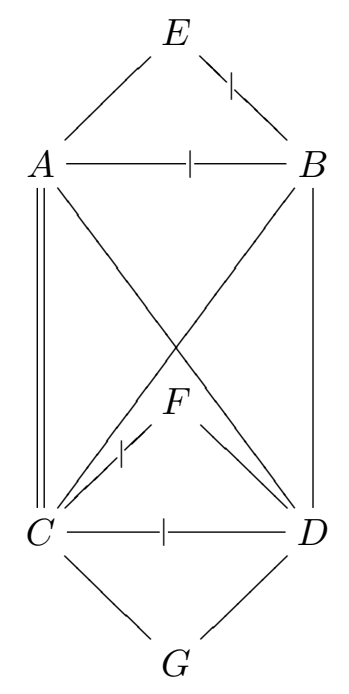

Theorem 6.2. There are c.e. sets $A$ and $B$ such that $A$ and $B$ have no infimum in the $<_{\text {st }}$ ordering, indeed, such $A$ and $B$ can be found with $A \not \equiv_{s t}$ $B$. There are c.e. sets $C$ and $D$ such that $C$ and $D$ have no supremum in the $<_{\text {st }}$ ordering, indeed, such $C$ and $D$ can be found with $C \not \equiv_{s t} D$.

Proof. We will construct $A, B, C, D, E, F$, and $G$ as described above, and $A$, $B, C$, and $D$ will be the desired sets. Since $E>_{s t} A$ and $E \ngtr_{s t} B, A \not \equiv{ }_{s t} B$. Since $A \nless_{s t} B$ and there is a gap between $A$ and $C$, and since $C<_{s t} A$ and $C<_{s t} B$, the only candidate for an $\inf$ of $A$ and $B$ is $C$. However, $D<_{s t} A$ and $D<_{s t} B$, but $D \nless_{s t} C$ and $D \not \equiv_{s t} C$ (since $F>_{s t} D$ but $F \ngtr_{s t} C$ ). So $A$ and $B$ have no inf. We've seen that $C \not_{s t} D$. Since $C \ngtr s t D$ and there is a gap between $A$ and $C$, and since $A>_{s t} C$ and $A>_{s t} D$, the only candidate for a sup of $C$ and $D$ is $A$. However $B>_{s t} C$ and $B>_{s t} D$, but $B \ngtr s t A$ and $B \not \equiv_{s t} A$, so $C$ and $D$ have no sup.

To construct the c.e. sets $A, B, C, D, E, F$ and $G$, we will use the methods of embedding the above partial ordering as in the proof of Theorem 3.1, but with additional requirements to ensure that there is a gap between $A$ and $C$. To do this, we will put restraint on $A$, as in the construction of a minimal set in $\S 5$. The restraint functions will be of the form $r(e, j, s)$, and we start off with $r(e, j,-1)=0$ for all $e, j$.

Stage s: To make $B \nless_{s t} E$. Let $l(E, s)$ be the total number of possible future entrants of $E$ declared up to this point in the construction. Let $\beta_{1}<\ldots<\beta_{l(E, s)}<n_{s}^{B}$ be greater than any number mentioned thus far in the construction. Enumerate $n_{s}^{B}$ into $B$, and declare that $\beta_{i}$ will enter $B$ when the $i$-th number less than $n_{s}^{B}$ enters $E$. There will be no restraint 
imposed on $B$, and we will never again declare anything less than $n_{s}^{B}$ to be eligible to enter $E$, so this will ensure that $m_{B}\left(n_{s}^{B}\right)>m_{E}\left(n_{s}^{B}\right)$.

To make $C \nless_{s t} F$, respecting $C<_{s t} A<_{s t} E$ and $C<_{s t} B$. Let $l(F, s)$ be the total number of possible future entrants of $F$ declared up to this point in the construction. Let $w_{i}(1 \leq i \leq l(F, s)), a_{j}^{w_{i}}(1 \leq i \leq l(F, s), 0 \leq j \leq s)$, $b_{j}^{w_{i}}(1 \leq i \leq l(F, s), 0 \leq j \leq s), e_{k}^{a_{j}^{w_{i}}}(1 \leq i \leq l(F, s), 0 \leq j, k \leq s)$, and $n_{s}^{C}$ all be distinct numbers greater than any number mentioned thus far in the construction. Moreover, the $w_{i}$ should all be less than $n_{s}^{C}$; the $a_{j}^{w_{i}}$ and $b_{j}^{w_{i}}$ should all be less than $w_{i}$; and the $e_{k}^{a_{j}^{w_{i}}}$ should all be less than $a_{j}^{w_{i}}$.

Enumerate $n_{s}^{C}$ into $C$, and declare that $w_{i}$ will enter $C$ when the $i$-th number less than $n_{s}^{C}$ enters $F$. There will be no restraint imposed on $C$, and we will never again declare anything less than $n_{s}^{C}$ to be eligible to enter $F$. This will ensure that $m_{C}\left(n_{s}^{C}\right) \geq m_{F}\left(n_{s}^{C}\right)$.

If at some later stage $t, \varphi_{j, t}\left(s\left(w_{i}, C\right)\right) \downarrow$, for a $j \leq s$, where $s\left(w_{i}, C\right)$ denotes the stage when (if ever) $w_{i}$ was enumerated into $C$, then enumerate $b_{j}^{w_{i}}$ into $B$ at stage $t$. This will help ensure that $B>_{s t} C$. We would also like to enumerate $a_{j}^{w_{i}}$ into $A$, but $A$ is subject to restraint, so we will deal with this later in the construction.

If at some later stage $t, \varphi_{k, t}\left(s\left(a_{j}^{w_{i}}, A\right)\right) \downarrow$, and if $k \leq s$, then enumerate $e_{k}^{a_{j}^{w_{i}}}$ into $E$ at stage $t$. This will help ensure that $E>_{s t} A$.

To make $G$ infinite, while respecting other requirements. Let $g, c_{i}^{g}(0 \leq$ $i \leq s), a_{j}^{c_{i}^{g}}(0 \leq i, j \leq s)$, and $e_{k}^{a_{j}^{c_{i}^{g}}}(0 \leq i, j, k \leq s)$ all be greater than any number mentioned so far in the construction, with $e_{k}^{a_{j}^{c_{i}^{g}}}<a_{j}^{c_{i}^{g}}<c_{i}^{g}<g(0 \leq$ $i, j, k \leq s)$.

Enumerate $g$ into $G$. If at some later stage $t, \varphi_{i, t}(s) \downarrow$, with $i \leq s$, then enumerate $c_{i}^{g}$ into $C$ and into $D$ at stage $t$. This will help ensure that $C>_{s t} G$ and $D>_{s t} G$. In this and later action we will continue to ensure that something is enumerated into $D$ only if at that same moment that same number is enumerated into $C$ (though not vice versa, because of the $F \ngtr s t$ requirement above).

If at some later stage $t, \varphi_{j, t}\left(s\left(c_{i}^{g}, C\right)\right) \downarrow$, with $i \leq s$, then enumerate $a_{j}^{c_{i}^{g}}$ into $B$ and $F$ at stage $t$. Since $c_{i}^{g}$ was enumerated into both $C$ and $D$, enumerating $a_{j}^{c_{i}^{g}}$ into $B$ will help ensure that $B>_{s t} C$, and $B>_{s t} D$, and enumerating $a_{j}^{c_{i}^{g}}$ into $F$ will help ensure that $F>_{s t} D$. We would also like to enumerate $a_{j}^{c_{i}^{g}}$ into $A$, but $A$ is subject to restraint, so we will deal with this later in the construction.

If at some later stage $t, \varphi_{k, t}\left(s\left(a_{j}^{c_{i}^{g}}, A\right)\right) \downarrow$, with $k \leq s$, then enumerate $e_{k}^{a_{j}^{c_{j}^{g}}}$ into $E$ at stage $t$. This will help ensure that $E>s t A$. 
To make $D \nless_{s t} C$. Let $l(C, s)$ be the total number of possible future entrants of $C$ declared up to this point in the construction. Let $\delta_{i}(1 \leq i \leq$ $l(C, s)), a_{j}^{w_{i}}(1 \leq i \leq l(C, s), 0 \leq j \leq s), e_{k}^{a_{j}^{w_{i}}}(1 \leq i \leq l(C, s), 0 \leq j \leq s, 0 \leq$ $k \leq s), n_{s}^{D}, a_{j}^{n_{s}^{D}},(0 \leq j \leq s), e_{k}^{a_{s}^{n_{s}^{D}}},(0 \leq j, k \leq s)$ all be distinct numbers greater than any number mentioned thus far in the construction. Moreover, the $\delta_{i}$ should all be less than $n_{s}^{D}$; the $a_{j}^{\delta_{i}}$ should all be less than $\delta_{i}$; and the $e_{k}^{a_{j}^{\delta_{i}}}$ should all be less than $a_{j}^{\delta_{i}}$. Also, the $e_{k}^{a_{j}^{n_{s}^{D}}}$ should all be less than the $a_{j}^{n_{s}^{D}}$ which should all be less than $n_{s}^{D}$.

Enumerate $n_{s}^{D}$ into both $C$ and $D$, and declare that $\delta_{i}$ will enter both $C$ and $D$ when the $i$-th number less than $n_{s}^{D}$ which is not of the form $\delta_{m}$ enters $C$. There will be no restraint imposed on $D$, and we will never again declare anything less than $n_{s}^{D}$ to be eligible to enter $C$ (except for the $\delta_{m}$ which we enumerate into $C$ only if we simultaneously enumerate it into $D$ ). This will ensure that $m_{D}\left(n_{s}^{D}\right)=m_{C}\left(n_{s}^{D}\right)$. We have also maintained our rule that we enumerate a number into $D$ only if we simultaneously enumerate the same number at the same moment into $C$.

If at some later stage $t, \varphi_{j, t}\left(s\left(\delta_{i}, D\right)\right) \downarrow$ with $j \leq s$, then enumerate $a_{j}^{\delta_{i}}$ into $B$ and $F$ at stage $t$. Since $\delta_{i}$ entered both $C$ and $D$, enumerating $a_{j}^{\delta_{i}}$ into $B$ will help ensure that $B>_{s t} C$, and $B>_{s t} D$, and enumerating $a_{j}^{\delta_{i}}$ into $F$ will help ensure that $F>_{s t} D$. We would also like to enumerate $a_{j}^{\delta_{i}}$ into $A$, but $A$ is subject to restraint, so we will deal with this later in the construction. Similarly, if $\varphi_{j, t}(s) \downarrow$, enumerate $a_{j}^{n_{s}^{D}}$ into $B$ and $F$ at stage $t$.

If at some later stage $t, \varphi_{k, t}\left(s\left(a_{j}^{\delta_{i}}, A\right)\right) \downarrow$, and if $k \leq s$, then enumerate $e_{k}^{a_{j}^{\delta_{i}}}$ into $E$ at stage $t$. Similarly if $\varphi_{k, t}\left(s\left(a_{j}^{n_{s}^{D}}, A\right)\right) \downarrow$, enumerate $e_{k}^{a_{j}^{n_{s}^{D}}}$ into $E$ at stage $t$. This will help ensure that $E>s t A$.

To make $A \nless_{s t} B$. Let $l(B, s)$ be the total number of possible future entrants of $B$ declared up to this point in the construction. Let $\alpha_{i}^{s}(1 \leq i \leq$ $l(B, s)), e_{j}^{\alpha_{i}^{s}}(1 \leq i \leq l(C, s), 0 \leq j \leq s)$, and $n_{s}^{A}$ all be distinct numbers greater than any number mentioned thus far in the construction. Moreover, the $\alpha_{i}$ should all be less than $n_{s}^{A}$, and the $e_{j}^{\alpha_{i}}$ should all be less than $\alpha_{i}$.

Enumerate $n_{s}^{A}$ into $A$ (it was chosen to be greater than any number mentioned so far, so in particular it is beyond all restraints.) When the $i$-th number less than $n_{s}^{A}$ is enumerated into $B$ we will declare that $\alpha_{i}^{s}$ desires to enter $A$, and this will remain the case until (if ever) $\alpha_{i}^{s}$ is enumerated into $A$. If for all $i \leq l(A, s)$, whenever $\alpha_{i}^{s}$ desires to enter $A$, it is eventually enumerated into $A$ (perhaps at a later stage than it first desired to be), we will have $m_{A}\left(n_{s}^{A}\right) \geq m_{B}\left(n_{s}^{A}\right)$, establishing that $A \nless_{s t} B$. 
If at a later stage $t, \varphi_{j, t}\left(s\left(\alpha_{i}^{s}, A\right)\right) \downarrow$, enumerate $e_{j}^{\alpha_{i}^{s}}$ into $E$ at stage $s$. This will help ensure that $E>_{s t} A$.

To make a gap between $A$ and $C$. We start off with $\tilde{r}(e, j)=r(e, j, s-1)$ for all $e, j \in \omega$, and let $\tilde{R}(j)=\max \{\tilde{r}(e, j) \mid e \leq j\}$.

Suppose $x$ is enumerated into $W_{e, s}$. If $x<\tilde{r}(e, j)$ for any $j$, reset $\tilde{r}(e, j)=$ $x$ for all such $j$. Otherwise, let $j$ be least such that $\tilde{r}(e, j)=0$, and reset $\tilde{r}(e, j)=x$.

Now, perform the following loop. Suppose $\varphi_{i, s}(s(x, C)) \downarrow$ for some $i, s$ such that $a_{i}^{x}$ was appointed. If $a_{i}^{x}>\tilde{R}(i)$, enumerate $a_{i}^{x}$ into $A_{s}$. For all $e, j$ with $a_{i}^{x}<\tilde{r}(e, j)$, reset $\tilde{r}(e, j)=0$. We define $\tilde{p}(t)$, the priority for $n_{t}^{A}$ to act, by induction. Let $\tilde{p}(0)=0$. Let $\tilde{p}(t+1)=\tilde{p}(t)$ if there is some $\alpha_{k}^{t}$ that desires to be enumerated such that $\alpha_{k}^{t} \leq \tilde{R}(\tilde{p}(t))$, and let $\tilde{p}(t+1)=\tilde{p}(t)+1$ otherwise. If $\alpha_{k}^{t}$ desires to be enumerated and $\alpha_{k}^{t}>\tilde{R}(\tilde{p}(t))$ then enumerate $\alpha_{k}^{t}$ into $A_{s}$, and for all $e, j$ with $\alpha_{k}^{t}<\tilde{r}(e, j)$, reset $\tilde{r}(e, j)=0$. If anything is enumerated, repeat the loop. Otherwise end the loop and set $r(e, j, s)=\tilde{r}(e, j)$ for all $e, j$, and $p(t, s)=\tilde{p}(t)$ for all $t$.

Note that as in the minimal set construction, the only way that $r(e, j, s)$ can increase as a function of $s$ is if the restraint is injured by enumeration into $A$ during a loop and set to 0 , and then reset to a larger number in a later stage. That is, we have $r(e, j, s+1) \leq r(e, j, s)$ unless $r(e, j, s)=0$. Also, if $r(e, j, s+1)>r(e, j, s)=0$, then this was because $r(e, j, s+1)$ was enumerated into $W_{e, s+1}$. Hence for any $e, j \in \omega, \lim _{s} r(e, j, s)<\infty$ or $\lim \sup _{s} r(e, j, s)=\infty$.

This completes the construction.

The facts that $E>_{s t} A, E \ngtr_{s t} B, B>_{s t} C, B>_{s t} D, F \ngtr_{s t} C, F>_{s t} D$, $C \ngtr s t D, C>_{s t} G$, and $D>_{s t} G$, follow exactly as in the embedding of the partial ordering in Theorem 3.1. We also easily see that $A \ngtr$ st $B$ since $E>_{s t} A$ and $E \ngtr_{s t} B$, and that $D \ngtr_{s t} C$ since $F>_{s t} D$ and $F \ngtr_{s t} C$.

It remains to show that there is a gap between $A$ and $C, A \nless_{s t} B$, $A>_{s t} D$, and $A>_{s t} C$. Note that since we only enumerate into $D$ if the same number is enumerated into $C$ at the same moment, $m_{C}(x) \geq m_{D}(x)$ for all $x$, so once we show $A>_{s t} C$, we immediately also have $A>_{s t} D$.

Lemma 6.3. $A>_{s t} C$.

Proof. It suffices to show that for all $i$, if $\varphi_{i}$ is total then there exists an $m$ such that whenever $a_{i}^{x}>m$ and $\varphi_{i, s}(s(x, C)) \downarrow$ then there is a stage $t \geq s$ where $a_{i}^{x}$ is enumerated into $A$.

For $e \leq i$, let $r(e, i)=\lim \sup _{s} r(e, i, s)$. Let $R(i)=\max \{r(e, i) \mid r(e, i)<$ $\infty \wedge e \leq i\}$. Suppose $a_{i}^{x}>R(i)$, and suppose $\varphi_{i, s}(x) \downarrow$. For $t \geq s$, let $b(t)=\min \{r(e, i, t) \mid r(e, i, t)>0 \wedge r(e, i)=\infty \wedge e \leq i\}$. We wish to show that there is a stage $t \geq s$ such that $b(t)$ is undefined, and hence $a_{i}^{x}$ would be enumerated into $A_{t}$. The function $b(t)$ cannot increase without first being undefined. So we may assume for a contradiction that $b=\lim _{t} b(t)$ exists. Let $t_{1}$ be such that $b=r\left(e, i, t_{1}\right)$ with $r(e, i)=\infty, e \leq i$, and $b(t)=b$ for 
all $t \geq t_{1}$. Since $\limsup _{t} r(e, i, t)=\infty$, there is some number less than $b$ enumerated into $A$ at a stage $t_{2}>t_{1}$. But then $b\left(t_{2}\right)$ is undefined.

Lemma 6.4. $A \nless_{s t} B$.

Proof. First note that the value of $p(t, s)$ can only change finitely often as $s$ increases (since there are finitely many $\alpha_{i}^{t^{\prime}}$ with $t^{\prime} \leq t$, and each can desire to enter $A$ and be enumerated at most once). Thus $p(t)=\lim _{s} p(t, s)$ exists for all $t$. Note also that $p$ is nondecreasing. We show by induction on $n$ that there exists a $t_{n}=\max \{t \mid p(t)=n\}$. Let $t_{-1}=-1$. Let $t>t_{n-1}$ be least such that all $\alpha_{i}^{t}>R(n)$, where $R(n)=\max \{r(e, n) \mid r(e, n)<\infty \wedge e \leq n\}$, as in Lemma 6.3. Then, as in the proof of Lemma 6.3, any $\alpha_{i}^{t}$ that desires to enter $A$ will eventually be enumerated into $A$ unless $p(t, s)>n$ for a.e. $s$. But this can only happen if there is some $t^{\prime}$ with $t_{n-1}<t^{\prime}<t$ such that every $\alpha_{i}^{t^{\prime}}$ that desires to enter $A$ eventually does. Hence $t_{n}$ exists, indeed $t_{n-1}<t_{n} \leq t$.

By definition of $t_{n}$, every $\alpha_{i}^{t_{n}}$ which desires to enter $A$ eventually does, hence for all $n, m_{A}\left(n_{t_{n}}^{A}\right) \geq m_{B}\left(n_{t_{n}}^{A}\right)$. Thus $A \nless_{s t} B$.

Lemma 6.5. For all $e$, if $W_{e}>_{s t} C$, then $A \ngtr_{s t} W_{e}$.

Proof. Case 1: For all $j \geq e, \lim _{s} r(e, j, s)<\infty$. Let $x_{j}=\lim _{s} r(e, j, s)$. Then either $W_{e}$ is finite, or there exist infinitely many $j$ such that $x_{j}<x_{j+1}$, and for all $j, m_{e}\left(x_{j}\right) \leq m_{A}\left(x_{j}\right)$ and hence $A \ngtr_{s t} W_{e}$.

Case 2: There is some $j$ such that $\lim \sup _{s} r(e, j, s)=\infty$. Let $j$ be the least such, let $r=\max \{r(e, i, s) \mid i<j\}$, and let $s^{\prime}$ be such that for any $\alpha_{i}^{t} \in A$ with $t \leq t_{e}$ we have $\alpha_{i}^{t} \in A_{s^{\prime}}$. By our conventions, if $\varphi_{i}$ is not total, then it converges on only finitely many $x$. Hence the function $f(x)=(\mu s)\left[(\forall i \leq j) \varphi_{i}(x) \downarrow \rightarrow \varphi_{i, s}(x) \downarrow\right]$ is computable. Let $y>r$ be any entrant of $C$, and let $x$ be the last entrant of $W_{e}$ less than or equal to $y$. If in addition $x \geq r$ and $x$ entered $W_{e}$ after stage $s^{\prime}$, we will have $x=r(e, k, s)$ for some $s>s^{\prime}$ and some $k \geq j$. Then, since $\lim \sup _{t} r(e, j, t)=\infty$, we know that $r(e, j, s) \leq r(e, k, s)=x$ will be injured. The only reason this would happen is because some $\varphi_{i}$ converged on the stage when something less than or equal to $y$ was enumerated into $C$. Thus $f\left(m_{C}(y)\right)>s=m_{e}(y)$. Since there are infinitely many such $y$, we see that $W_{e} \ngtr_{s t} C$.

Hence the sets $A, B, C, D, E, F$, and $G$ have the required properties.

\section{Super Domination}

To help simplify proofs in differential Geometry, Nabutovsky and Weinberger asked whether there exits a sequence $\left\{A_{i}\right\}$ of c.e. sets such that

$$
(\forall \text { computable } f)(\forall n)\left(\forall^{\infty} x\right)\left[m_{A_{i}}(x)>f\left(m_{A_{i+1}}(n x)\right)\right]
$$

Csima answered this question in her thesis [1] as follows.

Definition 7.1. Let $g$ be a computable function. For c.e. sets $A$ and $B$ we say $A>_{g-s t} B$ if for all computable functions $f$, for almost every $x$, 


$$
m_{A}(x)>f\left(m_{B}(g(x))\right) .
$$

Theorem 7.2. For any computable $g$, there exists a sequence $\left\{A_{i}\right\}$ of c.e. sets such that

$$
A_{i}>_{g-s t} A_{i+1}
$$

This answered the question of Nabutovsky and Weinberger using $g(x)=$ $x^{2}$.

However, this also raised the question of whether there are sets $A$ and $B$ such that $A>_{g-s t} B$ for all computable $g$.

Definition 7.3. For c.e. sets $A$ and $B$ we say $A$ strongly settling time dominates $B, A>_{s s t} B$, if for all computable functions $f$ and $g$, for almost every $x$,

$$
m_{A}(x)>f\left(m_{B}(g(x))\right) .
$$

Equivalently, $A>_{s s t} B$ if $A>_{g-s t} B$ for all computable $g$. The associated strict partial ordering on c.e. sets is denoted by $\mathcal{E}_{\text {sst }}$.

Note 7.4. For c.e. sets $A$ and $B, A>_{\text {sst }} B$ if and only if, for all strictly increasing computable functions $f$ and $g$, for almost every $x$,

$$
m_{A}(x)>f\left(m_{B}(g(x))\right) .
$$

Proof. If $f$ is computable, then there is a strictly increasing computable function $f^{*}$ with $f(x) \leq f^{*}(x)$. Note that for $f$ and $g$ computable, $B$ c.e., $f\left(m_{B}(g(x))\right) \leq f^{*}\left(m_{B}(g(x))\right) \leq f^{*}\left(m_{B}\left(g^{*}(x)\right)\right)$.

We will follow the conventions that if $\varphi$ is a partial computable function, then if $\varphi(n) \downarrow$ at stage $s$ then $\varphi(n)<s$, and for all $m<n, \varphi(m) \downarrow \leq \varphi(n)$.

Clearly $<_{s s t}$ implies $<_{s t}$, moreover, it is invariant under computable permutations and so remedies that "defect" in $\mathcal{E}_{s t}$. In fact, it respects not only 1-reducibility but even $w t t$-reducibility.

Theorem 7.5. The $<_{\text {sst }}$ ordering is well defined on wtt degrees. In fact, it respects wtt-reducibility in the sense that if $A \leq_{w t t} B<_{s s t} C$ or $A<_{s s t}$ $B \leq_{w t t} C$ then $A<_{s s t} C$.

Proof. We make use of the following lemma from Csima-Soare [2].

Lemma 7.6. Suppose $A \leq_{w t t} B$, with use bounded by $h$. Then there is a strictly increasing computable function $p$ such that

$$
(\forall x)\left[m_{A}(x) \leq p \circ m_{B}(h(x))\right] .
$$

Lemma 7.7. Let $A, B$, and $C$ be c.e. sets with enumerations $\left\{A_{s}\right\}_{s \in \omega},\left\{B_{s}\right\}_{s \in \omega}$, and $\left\{C_{s}\right\}_{s \in \omega}$, respectively. If $A \leq_{w t t} B$ with use bounded by $h$, and $B<_{s s t} C$ then $A<_{s s t} C$. 
Proof. We wish to show that for all strictly increasing computable functions $f$ and $g$, for a.e. $x, f\left(m_{A}(g(x))\right)<m_{C}(x)$. By Lemma 7.6, there is a strictly increasing function $p$ such that for all $x, m_{A}(x) \leq p\left(m_{B}(h(x))\right)$. So for all $x, f\left(m_{A}(g(x))\right) \leq f\left(p\left(m_{B}(h(g(x)))\right)\right)$. Since $B<_{s s t} C$, we have $p\left(f\left(m_{B}(h(g(x)))\right)\right)<m_{C}(x)$ for a.e. $x$. So for a.e. $x, f\left(m_{A}(g(x))\right)<m_{C}(x)$, as desired.

Lemma 7.8. Let $A, B$, and $C$ be c.e. sets with enumerations $\left\{A_{s}\right\}_{s \in \omega},\left\{B_{s}\right\}_{s \in \omega}$, and $\left\{C_{s}\right\}_{s \in \omega}$, respectively. If $A<_{s s t} B$ and $B \leq_{w t t} C$ then $A<_{s s t} C$.

Proof. We wish to show for all strictly increasing computable functions $f$ and $g$, for a.e. $x, f\left(m_{A}(g(x))\right)<m_{C}(x)$. By Lemma 7.6, there is a strictly increasing function $p$ such that for all $x, m_{B}(x) \leq p\left(m_{C}(x)\right)$. Since $A<s s t B$, $p\left(f\left(m_{A}(h(x))\right)\right)<m_{B}(x)$ for a.e. $x$. Now since $p$ is strictly increasing, this gives $f\left(m_{A}(g(x))\right)<m_{C}(x)$ for a.e. $x$ as desired.

These two lemmas show that $<_{s s t}$ respects $w t t$-reducibility and so is well defined on the $w t t$-degrees.

We first show that the relation $<_{s s t}$ is nontrivial.

Theorem 7.9. There exist c.e. sets $A$ and $B$ such $A>_{\text {sst }} B$ and $B$ is noncomputable.

Proof. We construct $A$ and $B$ to meet the requirements:

$R_{\langle i, j\rangle}: \varphi_{i}$ and $\psi_{j}$ total $\Rightarrow\left(\forall^{\infty} x\right) m_{A}(x)>\varphi_{i}\left(m_{B}\left(\psi_{j}(x)\right)\right)$.

$N_{e}: B \neq \Phi_{e}$.

Let $\Gamma_{l}$ be spread out so that between $\Gamma_{l}$ and $\Gamma_{l+1}$ there are guards $[i, j, k]_{l}$ for $0 \leq i, j, k \leq l$.

We will have numbers $x_{0}^{s}<x_{1}^{s}<\ldots$ which may be enumerated into $B$ for the sake of $N_{e}$.

Stage 0: Begin with $x_{m}^{0}=m$.

Stage $s+1$ : For the least $e$ for which $\Phi_{e}\left(x_{e}^{s}\right) \downarrow=0$ for some as yet unsatisfied $N_{e}$, enumerate $x_{e}^{s}$ into $B$ and declare $N_{e}$ satisfied.

If $\psi_{j}$ converges on $\Gamma_{l+2}$ for some $l \geq j$ for the first time at this stage, reset all $x_{e}^{s+1}$ to be greater than $s+1$ for unsatisfied $e \geq l$. Declare that the $[i, j, 0]_{l}$ will enter $A$ if $\varphi_{i}(s+1) \downarrow$. Let $t_{1}<\ldots<t_{n}$ denote the stages greater than $s$ when something less than or equal to $s+1$ enters $B$. Note that $n \leq l$. If we find at a later stage that $\varphi_{i}\left(t_{k}\right) \downarrow$, enumerate $[i, j, k]_{l}$ of $A$ at that stage.

Lemma 7.10. $\lim _{s \rightarrow \infty} x_{e}^{s}<\infty$.

Proof. $x_{e}^{s+1} \neq x_{e}^{s}$ only if some $\psi_{j}$ converged on $\Gamma_{l+2}$ for the first time at stage $s$, with $j \leq l \leq e$. This can only happen finitely often.

Lemma 7.11. The $N_{e}$ are satisfied.

Proof. Immediate since the $x_{e}^{s}$ reach a limit.

Lemma 7.12. The $R_{\langle i, j\rangle}$ are satisfied. 
Proof. Suppose $\varphi_{i}$ and $\psi_{j}$ are total.

It suffices to show that for a.e. $x$, whenever some $y \leq \psi_{j}(x)$ is enumerated into $B$ at a stage $s$ then some $z \leq x$ is enumerated into $A$ at a stage $t \geq \varphi_{i}(s)$.

Suppose $\Gamma_{l+1} \leq x<\Gamma_{l+2}$ for some $l \geq \max \{i, j\}$. By our conventions, $\psi_{j}(x) \leq \psi_{j}\left(\Gamma_{l+2}\right)$. At the stage when $\psi_{j}\left(\Gamma_{l+2}\right) \downarrow$, we appointed markers $[i, j, k]_{l}<\Gamma_{l+1} \leq x$ ready to enter $A$, one for each possible future entrant of $B$ below $\psi_{j}\left(\Gamma_{l+2}\right)$, and one in case there were no future entrants.

Proof. Thus $B$ is noncomputable and $A>_{s s t} B$ as required.

We now answer the question raised about $<_{s s t}$ in [2] that asks, in this stronger ordering, for the descending sequence requested by Weinberger and Nabutovsky for their work in differential geometry. In fact, we prove a bit more.

Theorem 7.13. Let $P$ be a computable partial ordering on $\mathbb{N}$ with no infinite ascending sequence. There exists a computable sequence $\left\{A_{n}\right\}$ of c.e. sets such that if $m<_{P} n$ then $A_{m}<_{s s t} A_{n}$.

Proof. We must meet the following requirements:

$$
R_{\langle i, j\rangle}^{\langle m, n\rangle}: m<_{P} n, \varphi_{i} \text { and } \psi_{j} \text { total } \Rightarrow\left(\forall^{\infty} x\right) m_{A_{n}}(x)>\varphi_{i}\left(m_{A_{m}}\left(\psi_{j}(x)\right)\right) .
$$

As in the previous theorem, we will be using markers and guards to help construct the sets. For each set $A_{n}$ that we build, we will use moveable markers $\Gamma_{l}^{n}$ with $l \geq n$, and between each $\Gamma_{l}^{n}$ and $\Gamma_{l+1}^{n}$ there will be guards of the form $[i, j, k]_{l}^{n}$ with $0 \leq i, j \leq l, 0 \leq k<g_{n}(l)$ to aid in meeting the $R$ requirements. Thus the number of guards between markers will be $(l+1)^{2} g_{n}(l)$, where $g_{n}(l)$ will be a uniformly computable function depending on $P$. The markers and their guards will be moved at most finitely often, and the only possible entrants of $A_{n}$ will be the guards and markers. We will maintain that if $k<n$ then $\Gamma_{k}^{n}<\Gamma_{l}^{n}$.

Each marker $\Gamma_{l}^{n}$ will have priority $\langle n, l\rangle$. So if some $\psi_{j}\left(\Gamma_{l+2}^{n}\right) \downarrow$ for $j \leq l$, markers of the form $\Gamma_{k}^{m}$ with $\langle m, k\rangle>\langle n, l\rangle$ and $m<_{P} n$ will be moved. This means that each marker will be moved only finitely often, so eventually will settle down. Also, each marker $\Gamma_{l}^{n}$ will only have finitely many markers (and hence finitely many possible entrants of sets) that it can't move, and so (since it knows about them in advance) can ensure that there are enough guards between $\Gamma_{l}^{n}$ and $\Gamma_{l+1}^{n}$ to accommodate them.

We now define a computable function $h(\langle m, k\rangle)$ bounding the number of times marker $\Gamma_{k}^{m}$ might be moved. We let $h(\langle m, k\rangle)=0$ if $m \nless_{P} n$ for all $\langle n, l\rangle\left\langle\langle m, k\rangle\right.$, since in such a situation $\Gamma_{k}^{m}$ will never be moved. We then inductively define

$$
h(\langle m, k\rangle)=\sum_{\langle n, l\rangle<\langle m, k\rangle \wedge m<{ }_{P} n}(h(\langle n, l+2\rangle)+1)(l+1),
$$


since the marker $\Gamma_{k}^{m}$ can be moved only if $\psi_{j}\left(\Gamma_{l+2}^{n}\right) \downarrow$, where $j \leq l,\langle n, l\rangle<$ $\langle m, k\rangle$ and $m<_{P} n$. Note that $h$ is computable since $P$ has no infinite ascending chain.

Now that we have $h(\langle m, k\rangle)$, we are ready to define the uniformly computable $g_{n}(l)$. The function $g_{n}(l)$ represents the total number of possible entrants of higher priority that can't be moved when a convergence on $\Gamma_{l+2}^{n}$ is observed, plus the number of times $\Gamma_{l+2}^{n}$ might move. For each $\Gamma_{k}^{m}$ that can't be moved, there are $(k+1)^{2} g_{m}(k)$ possible entrants, and $\Gamma_{k}^{m}$ might move $h(\langle m, k\rangle)$ many times. Note that if $\langle m, k\rangle=\langle n, l\rangle$ then $m=n$ and $m \nless P n$. So we inductively define

$$
g_{n}(l)=h(\langle n, l+2\rangle)+\sum_{\langle m, k\rangle<\langle n, l\rangle \text { and } m<_{P} n}\left((k+1)^{2} g_{m}(k)\right) h(\langle m, k\rangle) .
$$

Stage 0: Spread out the markers $\Gamma_{l}^{n}$ such that there is room for the $(l+1)^{2} g_{n}(l)$ many guards of the form $[i, j, k], 0 \leq i, j \leq l, 0 \leq k<g_{n}(l)$ between $\Gamma_{l}^{n}$ and $\Gamma_{l+1}^{n}$. Also ensure that all markers and guards are distinct, so at most one $\psi_{j}\left(\Gamma_{l+2}^{n}\right) \downarrow$ at any given stage.

Stage $s+1$ : If, for $j<l, \psi_{j, s}\left(\Gamma_{l+2}^{n}[s]\right) \downarrow$, but $\psi_{j, s-1}\left(\Gamma_{l+2}^{n}[s]\right) \uparrow$, we say $(n, l, j)$ receives attention at stage $s+1$. Reset all $\Gamma_{k}^{m}$ with $\langle m, k\rangle>\langle n, l\rangle$ and $m<_{P} n$ which have already been defined, and their corresponding guards, to all be greater than $s+1$. That is, if a marker $\Gamma_{k}^{m}$ is moved, the guards between $\Gamma_{k-1}^{m}$ and $\Gamma_{k}^{m}$ should all be moved. They should be moved far enough that all the various markers and guards are on distinct numbers not yet enumerated.

Let $a$ be the number of previous stages where $(n, l, j)$ received attention. Note that $0 \leq a<h(\langle n, l+2\rangle)$. Declare that the $[i, j, a]_{l}^{n}$ will enter $A_{n}$ if $\varphi_{i}(s+1) \downarrow$. That is, at the stage $t$ when $\varphi_{i}(s+1)$ converges, enumerate $[i, j, a]_{l}^{n}[t]$ into $A_{n}$.

General Declaration: Recall that $g_{n}(l)-h(\langle n, l+2\rangle)$ represents the total number of possible entrants of higher priority that can't be moved when a convergence on $\Gamma_{l+2}^{n}$ is observed. Let $s_{1}<\ldots<s_{r}$ denote the stages, $s$, with the following property. There is some $j \leq l$ and $t<s$ such that $\psi_{j, t}\left(\Gamma_{l+2}^{n}[t]\right) \downarrow=\psi_{j, s}\left(\Gamma_{l+2}^{n}[s]\right) \downarrow, \Gamma_{l+2}^{n}[t]=\Gamma_{l+2}^{n}[s]$, and something of the form $[a, b, c]_{k}^{m}$ less than $\psi_{j}\left(\Gamma_{l+2}^{n}[s]\right)$ enters $A_{m, s}$ for some $\langle m, k\rangle<\langle n, l+2\rangle, m<_{P}$ $n$. Note that $r \leq g_{n}(l)-h(\langle n, l+2\rangle)$. If we find at some stage $t^{\prime}$ that for some $i \leq l, \varphi_{i}\left(s_{k}\right) \downarrow$, enumerate $[i, j, h(\langle n, l+1\rangle)+k-1]_{l}^{n}\left[t^{\prime}\right]$ into $A_{n}$ at stage $t^{\prime}$.

Lemma 7.14. The $\Gamma_{l}^{n}$ reach a limit.

Proof. We have already computed that $\Gamma_{l}^{n}$ can move at most $h(\langle n, l\rangle)$ times.

Lemma 7.15. The $R_{\langle i, j\rangle}^{\langle m, n\rangle}$ are satisfied.

Proof. Suppose $\varphi_{i}$ and $\psi_{j}$ are total and $m<_{P} n$. 
It suffices to show that for a.e. $x$, whenever some $y \leq \psi_{j}(x)$ is enumerated into $A_{m}$ at a stage $s$ then some $z \leq x$ is enumerated into $A_{n}$ at a stage $t \geq \varphi_{i}(s)$.

Suppose $\Gamma_{l+1}^{n} \leq x<\Gamma_{l+2}^{n}$ for some $l \geq \max \{i, j\}$. By our conventions, $\psi_{j}(x) \leq \psi_{j}\left(\Gamma_{l+2}^{n}\right)$. Suppose $y \leq \psi_{j}(x)$ was enumerated into $A_{m}$ at stage $s$. Then $y \leq \psi_{j}\left(\Gamma_{l+2}^{n}\right)$. Case 1: $y \leq \psi_{j, s}\left(\Gamma_{l+2}^{n}[s]\right) \downarrow$. In this case, a marker $[i, j, k]_{l}^{n}[t]<\Gamma_{l+1}^{n}[t] \leq \Gamma_{l+1}^{n} \leq x$ was enumerated into $A_{n}$ at the stage $t$ when $\varphi_{i}(s)$ converged. Case $2: \psi_{j, s}\left(\Gamma_{l+2}^{n}[s]\right) \uparrow$ or $y>\psi_{j, s}\left(\Gamma_{l+2}^{n}[s]\right) \downarrow$. In this case, there must have been a stage $t>s$ such that $y \leq \psi_{j, t}\left(\Gamma_{l+2}^{n}[t]\right) \downarrow$, and at stage $t$, we would have had a number $[i, j, a]_{l}^{n}\left[t^{\prime}\right] \leq \Gamma_{l}^{n}\left[t^{\prime}\right] \leq \Gamma_{l}^{n} \leq x$ which would be enumerated into $A_{n}$ at the stage $t^{\prime}$ when $\varphi_{i}(t)$ converged, which by our conventions was after the stage when $\varphi_{i}(s)$ converged.

This completes the proof of the theorem.

Now although Theorem 7.13 is phrased in terms of a partial ordering $P$, we have not done anything to preserve the nonordering facts in $P$. Thus essentially what is proven is that every countable linear ordering with no infinite ascending sequences (i.e. those of the form $\alpha^{*}$ for a countable ordinal $\alpha$ ) can be embedded in $\mathcal{E}_{\text {sst }}$. We expect that further work along these lines should extend the result to all linear orderings. Adding the requirements to preserve nonorder needs additional types of arguments. We might also expect that the stronger result that makes $A_{i} \not_{T} A_{j}$ if $i \nless_{P} j$ and $i \neq j$ is also true. Csima and Soare do this for the linear ordering $\mathbb{Z}$ in $[2]$ and a tree argument should produce the same extension of Theorem 3.1 embedding every countable ordering in $\mathcal{E}_{s t}$. We thus close with a conjecture.

Conjecture 7.16. For every countable partial ordering $P$ there is an embedding into the c.e. sets taking $i \in P$ to $A_{i}$ that is order preserving with respect to both $<_{s s t}$ and $<_{T}$, i.e. if $i<_{P} j$, then $A_{i}<_{s s t} A_{j}$ (and so, $a$ fortiori, $A_{i} \leq_{T} A_{j}$ ) and if $i \nless_{P} j$ and $i \neq j$, then $A_{i} \not_{T} A_{j}$ (and so, $a$ fortiori, $A_{i} \nless_{s s t} A_{j}$ ). (Note that if $i \neq j$, then either $i \nless_{P} j$ or $j \nless_{P} i$ and so $A_{i} \neq_{T} A_{j}$.)

\section{REFERENCES}

[1] B. F. Csima, Applications of Computability Theory to Prime Models and Differential Geometry, Ph.D. thesis, The University of Chicago, 2003.

[2] B. F. Csima and R. I. Soare, Computability Results Used in Differential Geometry, Journal of Symbolic Logic, to appear.

[3] A. Nabutovsky and S. Weinberger, The Fractal Nature of Riem/Diff I, Geometrica Dedicata 101 (2003), 1-54.

[4] H. Rogers, Jr., Theory of Recursive Functions and Effective Computability, McGrawHill, New York, 1967.

[5] R. I. Soare, Recursively Enumerable Sets and Degrees: A Study of Computable Functions and Computably Generated Sets, Springer-Verlag, Heidelberg, 1987.

[6] R. I. Soare, Computability theory and differential geometry, Bull. Symb. Logic 10 (2004), 457-486. 
[7] R. I. Soare, Computability Theory and Applications, Springer-Verlag, Heidelberg, to appear.

[8] S. Weinberger, Computers, rigidity and moduli. The large scale fractal geometry of Reimannian moduli space, M.B. Porter Lectures, Princeton UNiversity Press, Princeton NJ, 2005

Department of Pure Mathematics, University of Waterloo, Waterloo, On, CANADA N2L $3 \mathrm{G} 1$

$U R L$ : www.math.uwaterloo.ca/ csima

E-mail address: csima@math.uwaterloo.ca

Department of Mathematics, Cornell University, Ithaca NY 14853

URL: http://www. math. cornell.edu/ shore/

E-mail address: shore@math.cornell.edu 